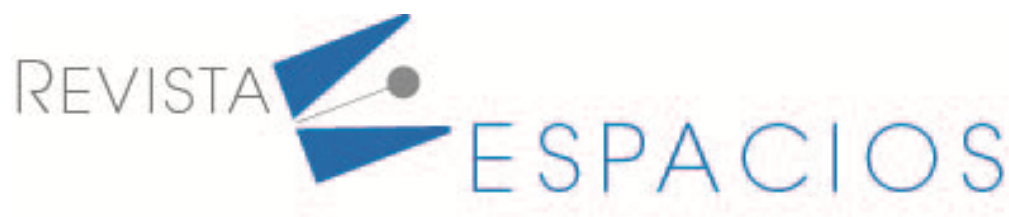

Vol. 42 (06) $2021 \cdot$ Art. 5

Recibido/Received: 04/12/2021 • Aprobado/Approved: 20/02/2021 • Publicado/Published: 30/03/2021

\title{
La economía colaborativa, sus beneficios y efectos
}

\section{Sharing economy and its benefits and its effects}

\author{
CORONEL, Andrés A. ${ }^{1}$ \\ ORTEGA, Manuel O. ${ }^{2}$ \\ SCALDAFERRI, Walter F. ${ }^{3}$
}

\section{Resumen}

Este artículo tiene el objetivo de analizar la economía colaborativa y cómo este concepto está influyendo en la economía de varios países, en especial en los de vías de desarrollo como Ecuador. Las plataformas digitales, para las cuales la economía colaborativa se creó, están creciendo en transacciones y ventas beneficiando así a parte de la población, pero al mismo tiempo hay dudas del verdadero impacto de este sistema. Este estudio es exploratorio y basado en referencias bibliográficas de otros autores. Palabras claves: economía colaborativa, plataformas tecnológicas, innovación

\begin{abstract}
This article has the objective of analyze the sharing economy and how this concept is influencing the economy in many countries specially developing countries like Ecuador. The digital platforms that are used in the sharing economy are growing in number of transactions and sales, giving benefit to part of the population, but at the same time, there are doubts of the real impact of this system. This is an exploratory study based on bibliographical references of other authors.

Keywords: Sharing economies, technological platforms, innovation
\end{abstract}

\section{Introducción}

La economía colaborativa es un concepto que está ganando cada vez más fuerza. Se trata del intercambio de bienes y servicios que se realiza a través de plataformas digitales (Alfonso Sánchez, 2016). A nivel mundial se han desarrollado distintas aplicaciones o sitios web y éstos son utilizados en varios países del mundo. En latinoamérica y en Ecuador tenemos varios ejemplos de estas plataformas, tales como Uber, Cabify, Airbnb, Glovo, Rappi, entre otras.

Para comprender mejor las economías colaborativas explicamos el ejemplo de Airbnb. Esta plataforma, creada en Estados Unidos, pone a disposición el servicio de hospedaje como alternativa a la hotelería. Por un lado hay personas que ofrecen habitaciones, suites, departamentos o incluso casas enteras, colocando la información en la web y, por el otro lado, están quienes buscan alternativas de hospedajes que se ajusten a sus requerimientos

\footnotetext{
${ }^{1}$ Docente. Instituto Superior Tecnológico Blue Hill College. acoronel@bluehill.edu.ec

${ }^{2}$ Docente. Instituto Superior Tecnológico Blue Hill College. mortega@bluehill.edu.ec

${ }_{3}^{3}$ Docente. Instituto Superior STecnológico Blue Hill College. wscaldaferri@bluehill.edu.ec
} 
de presupuesto, ubicación y otros. En un estudio realizado en España y Portugal se evidenció el crecimiento en el uso de esta plataforma como alternativa de hospedaje (Palos-Sánchez \& Correia, 2018).

En Ecuador es muy común el uso de las aplicaciones para entregas a domicilio, tales como Glovo, Uber Eats, Rappi y Picker, así como Apps para movilización como Cabify y Uber. De este tipo de aplicaciones es que se pretende realizar el respectivo análisis en cuanto el impacto sobre el empleo, además del apoyo indirecto a la economía.

\section{Metodología}

Este estudio se basa en una metodología exploratoria o interpretativa, mediante la cual se toman como referencia diferentes fuentes bibliográficas de autores relacionados con economía colaborativa, así como fuentes estadísticas respectivas al empleo y la economía. Este nuevo sector de la economía, el cual pretendemos estudiar, es relativamente nuevo y por lo tanto no existe mucha información sobre el tema. En el caso particular de Ecuador y Latinoamérica, el término de economía colaborativa no contiene muchas fuentes de referencia bibliográfica. Como propone Morales (2014) la idea en una investigación exploratoria es poder formular un problema de investigación. El abanico en cuanto a lo ofrecido por las plataformas digitales es tan amplio, que se puede partir de muchas ramas de investigación. La información estadística analizada corresponde mayormente a Ecuador y alguna otra cifra global. Esta investigación no cuenta con datos cualitativos o cuantitativos. En una segunda parte se plantea realizar trabajo en campo para ahondar en este tema.

\section{Resultados}

El concepto de economía colaborativa ha traído consigo la creación de nuevas empresas de base tecnológicas y de esa manera una nueva forma de consumo. La economía colaborativa a la vez presenta un reto en muchos aspectos tales como el tributario (Antón, Iñaki, \& Estrada, 2016). Sin embargo, así como comporta desafíos, también es importante analizar los beneficios que acarrean las plataformas digitales de la también llamada economía compartida.

Ecuador al igual que varios países en América Latina, ha venido experimentando recesiones en sus economías. Actualmente y debido al confinamiento experimentado a causa del Covid-19, muchos negocios y empresas han cerrado o reducido su producción/operación. Según un reporte del Banco Mundial emitido en junio pasado, se proyecta que la economía mundial caiga en un $-5.2 \%$, la economía Latinoamericana estaría en $-7.2 \%$ y la economía ecuatoriana prevé una reducción de su PIB -7.4\% (World Bank, 2020).

Tabla 1

Proyecciones de crecimiento del PIB

\begin{tabular}{ccc}
\hline Territorio & $2020 \mathrm{p}$ & $2021 \mathrm{p}$ \\
\hline Mundial & $-5.2 \%$ & $4.2 \%$ \\
Latinoamérica y el Caribe & $-7.2 \%$ & $2.8 \%$ \\
Ecuador & $-7.4 \%$ & $4.1 \%$ \\
\hline
\end{tabular}

Fuente: World Bank. (2020, June).

Nota: $p=$ proyección

Ese decrecimiento en la producción de los países está provocando un alto número de desempleo para sus ciudadanos. De acuerdo con unas cifras presentadas por el Instituto Ecuatoriano de Estadísticas y Censos, entre los meses de mayo y junio del 2020 el desempleo en Ecuador alcanzó un nivel del 13.3\% (Instituto Nacional de Estadísticas y Censos, INEC, 2020) cuando en junio del 2019 era tan solo del 4.4\%. Es aquí donde el empleo que genera la economía colaborativa juega su rol. 
Según el último reporte del Global Entrepreneurship Monitor Ecuador (GEM 2019) citado por Lasio (2020), no hay cifras exactas del impacto de la economía colaborativa en este tiempo de pandemia, pero indica que durante la emergencia sanitaria las aplicaciones de logísticas, en especial las de entregas, tuvieron un crecimiento importante No obstante, el mismo documento muestra un cuadro comparativo en donde Ecuador está por debajo de otros países en lo que corresponde a la participación de la economía colaborativa (Lasio, 2020)

Tabla 2

\begin{tabular}{cc} 
Participación en economía colaborativa \\
\hline País & $\begin{array}{c}\% \text { de población } \\
18-64 \text { años }\end{array}$ \\
\hline Ecuador & 0.5 \\
Argentina & 1 \\
Chile & 2.9 \\
Uruguay & 2 \\
\hline \multicolumn{2}{r}{ Fuente: Lasio. (2020) } \\
Nota: Los datos corresponden al año 2019
\end{tabular}

En un reportaje del diario El Universo se reportó un estudio del impacto económico de Glovo en el Ecuador en su primer año de operaciones. El artículo muestra cifras interesantes como una ganancia promedio de USD.3.50/hora para los repartidores, lo que genere un ingreso mensual entre USD. 569.00 y USD. 1,736.00, según las horas y días dedicados a la actividad (García, 2019). Estos ingresos superan los de un salario básico unificado. Por lo tanto, para varias personas se ha vuelto atractivo esta modalidad de trabajo.

\subsection{Beneficios de la economía colaborativa}

Gracias a la economía colaborativa, las personas pueden generar ingresos que les permita tener un extra para su presupuesto o en otros casos dedicarse tiempo completo a alguna actividad relacionada con una de las plataformas digitales descritas. En tiempos de recesiones económicas, donde las tasas de desempleo están al alta, el poder contar con alternativas de ingresos como las que se obtienen a través de las plataformas digitales de economía colaborativa es un alivio para muchas familias.

De igual manera, gracias a varias de estas aplicaciones, en especial las de servicios de entrega, muchos negocios han podido incrementar sus ventas, llegando a más consumidores, de manera que en algunos sectores se ha dinamizado un poco más la economía. Durante la pandemia, gracias a Glovo, Uber eats, Rappi y demás aplicaciones, varios negocios pudieron sobrellevar la crisis y mantenerse en el mercado.

En el reporte del GEM 2019 se menciona el término GIG Economy que son plataformas digitales que ofrecen servicios profesionales de personas para algún proyecto puntual (Lasio, 2020). El GIG Economy y la economía colaborativa están estrechamente relacionadas y cada vez crean no solo nuevos hábitos para los consumidores, sino también nuevas oportunidades de ingresos.

Estos conceptos no solo aplican para servicios a domicilios, taxis u hospedajes como lo son Glovo, Uber y Airbnb respectivamente. Existe una infinidad de aplicaciones utilizadas en otros países, y que podrían ser utilizadas en el país como forma de contrarrestar el desempleo. Hay aplicaciones para compartir viajes en carro como Bla Bla Car en Europa. Otro ejemplo es Fiver en Estados Unidos que conecta a personas que buscan páginas web, fotografías y artes gráficas con desarrolladores de páginas, fotógrafos y diseñadores. Incluso existen Apps para los amantes caninos en donde se puede conseguir una persona que pasee a su perro o que lo cuide mientras se va de viaje.

A partir de septiembre del 2020, el Servicio de Rentas Internas ha decidido gravar con el Impuesto de Valor Agregado (IVA) a los servicios digitales. Dentro de estos servicios digitales se encuentran las plataformas de 
economía colaborativa. Por lo tanto, su uso también contribuye a una recaudación fiscal para el gobierno ecuatoriano.

\subsection{Efectos de la economía colaborativa a considerar}

La economía colaborativa puede ser muy beneficiosa en materia de empleo. Sin embargo, algunos autores han manifestado preocupaciones con respecto a este nuevo sector de la economía.

A pesar de no tratarse de un emprendimiento o de negocios informales, varias de las plataformas digitales no están constituidas como empresas en los diferentes países donde tienen operación. De tal manera, las personas que se benefician prestando sus servicios no cuentan con ningún beneficio de ley o seguro social. Asimismo, en el caso de los hospedajes ofrecidos en plataformas como Airbnb, no se cuenta con ningún tipo de permiso de funcionamiento. Los negocios informales se convierten en competencia desleal para los negocios que sí cumplen y pagan todo lo que la ley exige (Corone \& Ortega, 2019). El mismo ejemplo para el hospedaje se puede aplicar para las plataformas para movilidad de personas como Uber o Cabify versus los taxis regulados por las agencias de tránsito.

Además de lo expuesto, al romperse la relación directa entre empresario y cliente, existe un riesgo en garantizar el derecho al consumidor (Martín, 2017). Otra preocupación que genera la economía colaborativa radica en la posibilidad de que sea una nueva forma de explotación laboral (Reygadas, 2018). Al tratarse de un nuevo segmento o también un nuevo sector de la economía relacionado con tecnología e innovación, muchos países carecen de las regulaciones respectivas para hacer respetar ciertas leyes mínimas necesarias para salvaguardar a una sociedad. Por lo tanto, quedan dudas si las plataformas digitales se aprovechan de los vacíos legales de las naciones en donde se encuentran operando para aprovecharse de esa situación (Bó \& Petrini, 2019).

Es importante evaluar a detenimiento cada una de esas cuestiones que surgen con este nuevo modelo tecnológico, que ciertamente brinda beneficios, pero del cual hay que tener cuidado de sus efectos. Aún no sabemos si el balance será positivo o negativo. Aun así, queda una reflexión, ċlas nuevas tecnologías se deben adaptar a las leyes y regulaciones actuales o las leyes y regulaciones son las que se tienen que adaptar a las nuevas tendencias tecnológicas?

\section{Conclusiones}

La economía colaborativa, basada en el intercambio de bienes y servicios a través de plataformas digitales, está jugando un rol importante en medio de una crisis económica y laboral debido a la pandemia mundial, creando un nuevo mercado laboral y salvando a muchos negocios de la quiebra. Este éxito dado a la gran demanda debido al confinamiento atrae a más plataformas ofreciendo otros bienes y servicios no tan convencionales, pero que de igual forma tienen acogida, creando así puestos de trabajos con salarios un poco arriba de la escala normal, pero que a su vez no tienen el beneficio de las leyes laborales, ni tampoco la seguridad social.

Quedan dudas si las plataformas digitales están tomando ventaja de este vacío legal hasta que se aplique una ley para ellos, o si la ley a aplicarse sería igual a la aplicada a las empresas normales o se adaptaría una nueva forma de ley a este tipo de economía.

\section{Referencias bibliográficas}

Alfonso Sánchez, R. (2016). Economía colaborativa: un nuevo mercado para la economía social. www.ciriec.eswww.ciriec-revistaeconomia.es

Antón, Á., Iñaki, A., \& Estrada, B. (2016). El consumo colaborativo en la era digital: Un nuevo reto para la fiscalidad. 
Bó, G. J. D., \& Petrini, M. (2019). Empowering and resisting in a sharing economy: Two sides of the same coin. BAR - Brazilian Administration Review, 16(3). https://doi.org/10.1590/1807-7692BAR2019180133

Corone, A., \& Ortega, M. (2019). Perspectivas del emprendimiento en el Ecuador, sus dificultades y la informalidad. Yachana, Revista Científica, 8(3).

García, J. (2019, October 22). Glovo cumple un año y presenta estudio sobre impacto económico de la APP | Doctor Tecno | La Revista | El Universo. Retrieved October 18, 2020, from Diario El Universo website: https://www.eluniverso.com/larevista/2019/10/22/nota/7570457/glovo-cumple-ano-presenta-estudiosobre-impacto-economico-app

Instituto Nacional de Estadísticas y Censos (INEC). (2020). Encuesta Nacional de Empleo, Desempleo y Subempleo Telefónica Indicadores laborales Mayo-junio 2020. https://www.ecuadorencifras.gob.ec/documentos/webinec/EMPLEO/2020/ENEMDU_telefonica/Principal es_Resultados_Mercado_Laboral.pdf

Lasio V; Amaya A; Zambrano J; Ordeñana X. (2020). Global Entrepreneurship Monitor. Ecuador

Martín, M. (2017). Economía Colaborativa y protección del consumidor. Universidad de Valladolid. Revista de Estudios europeos, Nro. 70, recuperado de https://dialnet.unirioja.es/descarga/articulo/6258557.pdf

Morales, N. (2014). Investigación Exploratoria: Tipos, Metodología y Ejemplos. https://www.mendeley.com/catalogue/e4fe5d08-9354-3dfe-ad3a-fb923cb8e7b5/

Palos-Sanchez, P. R., \& Correia, M. B. (2018). The collaborative economy based analysis of demand: Study of airbnb case in Spain and Portugal. Journal of Theoretical and Applied Electronic Commerce Research, 13(3), 85-98. https://doi.org/10.4067/S0718-18762018000300105

Reygadas, L. (2018, April). Dones, falsos dones, bienes comunes y explotación en las redes digitales. Diversidad de la economía virtual. Retrieved April 11, 2020, from http://www.scielo.org.mx/scielo.php?script=sci_arttext\&pid=S1607-050X2018000100070\&lang=es

World Bank. (2020, June). Global Economic Prospects. Retrieved October 11, 2020, from https://www.bancomundial.org/es/publication/global-economic-prospects

Esta obra está bajo una Licencia Creative Commons Attribución-NoCommercial 4.0 International

\section{(cc) EY-NO}

